\title{
Auto-Generation and Auto-Tuning of 3D Stencil Codes on GPU Clusters .
}

\author{
Yongpeng Zhang, Frank Mueller \\ North Carolina State University, Raleigh, NC 27695-7534 \\ muller@cs.ncsu.edu
}

\begin{abstract}
This paper develops and evaluates search and optimization techniques for auto-tuning 3D stencil (nearest-neighbor) computations on GPUs. Observations indicate that parameter tuning is necessary for heterogeneous GPUs to achieve optimal performance with respect to a search space. Our proposed framework takes a most concise specification of stencil behavior from the user as a single formula, auto-generates tunable code from it, systematically searches for the best configuration and generates the code with optimal parameter configurations for different GPUs. This auto-tuning approach guarantees adaptive performance for different generations of GPUs while greatly enhancing programmer productivity. Experimental results show that the delivered floating point performance is very close to previous handcrafted work and outperforms other auto-tuned stencil codes by a large margin.
\end{abstract}

\section{INTRODUCTION}

Main-stream microprocessor design no longer delivers performance boosts by increasing the processor clock frequency due to power and thermal constraints. Nonetheless, advances in semiconductor fabrication still allow the transistor density to increase at the rate of Moore's law. This has resulted in the proliferation of manycore parallel architectures and accelerators, among which GPUs quickly established themselves as suitable for applications that exploit fine-grained data-parallelism.

Still, software development for parallel architectures turns out to be more difficult than that for uni-processors in terms of obtaining high performance, even when aided by new programming models such as CUDA [1] and OpenCL [9]. Programmers spend substantial time and effort to understand the underlying architecture to best utilize all resources. This can become a daunting task since performance is affected by a multitude of architectural features. Even worse, architectural difference between generations of the same hardware line may require a diversity of optimization strategies with sometimes opposite optimal set-points. Programmers may

${ }^{*}$ This work was supported in part by NSF grants 1058779 , 0958311, 0937908, and DOE grant DE-FG02-08ER25837. have to explore many (if not all) combinations of optimization options and parameter values to determine the best configuration for a particular hardware. This poses a great challenge since programmer productivity is adversely affected by lengthy tuning efforts. Simply re-profiling and re-writing the program each time the hardware is upgraded is neither desirable nor feasible over time.

Current compilers for general-purpose languages struggle to balance portability, performance and programmability. Domainspecific languages (DSLs), in contrast, offer a promising solution at the expense of sacrificing language generality [3]. DSLs have restricted expressiveness aimed at a particular domain. It is precisely this domain-specific knowledge that allows the DSL-compiler to attain performance achieve comparable to hand-coded domain implementations. In contrast, general-purpose languages are inherently limited in their optimization scope in exchange for assuring correctness and good overall (but not best) performance on average for a wide range of applications. Examples of well-known DSLs are HTML for web pages, Matlab for scientific computation and SQL for database queries.

This work focuses on providing a portable source-to-source autogeneration and auto-tuning framework for iterative 3D Jacobi stencil computations on different GPUs. We generate stencil code as native CUDA code for NVIDIA GPUs, yet the same principles apply for GPUs of other vendor and comparable programming models, e.g., OpenCL [9].

Stencil (nearest-neighbor) computations are widely used in scientific computing, including structured grids as well as implicit and explicit partial differential equation (PDE) solvers in domain ranging from thermo/fluid dynamics over climate modeling to electromagnetics among others. An iterative explicit stencil computation is comprised of computation-intensive kernel. At each discrete timestep, all stencil points are updated according to values of their spatial neighbors from a previous timestep. On one hand, the uniform and communication-free behavior is well suited for the SIMT (single instruction multiple threads) paradigm advocated by stateof-the-art GPUs. On the other hand, an efficient GPU implementation is sensitive to neighbors accessing patterns across different stencils. One key characteristic of most stencil computations is the overlap in input values to update multiple neighboring points. Exploiting this property is crucial to achieve competitive performance on GPUs. One common GPU technique is to use the on-chip shared memory (shared by a warp/block of threads) as an intermediate storage space for overlapped input values. Instead of letting each thread fetching all inputs from off-chip global memory, all inputs are first cooperatively loaded to shared memory before they are referenced 
when computing a new stencil value. This is beneficial even in more recent generations of cache-enabled GPUs since this shared memory is orders of magnitude faster than global memory. It is critical to determine how many threads should be grouped together in one block: Increasing the block size increases shared memory data reuse but may also deteriorate the GPU's occupancy rate of processing units [1].

There are many other factors that affect the performance. For example, how many stencil points should a thread work on? The larger the number, the more instruction-level optimizations can be applied by a compiler. But the less data-parallelism is exposed, the higher risk is for not fully utilizing a GPU's processing units. Also, is mapping inputs to texture memory faster? Our experiments show that the answer varies from case to case. Overall, there is no universal, optimal configuration for all types of stencil computations on different GPU models. Therefore, auto-tuning is not only desirable but also necessary to improve performance in this particular domain.

This work falls into the area of implicitly parallel programming models [10]. Our model relies on a compiler to generate highly efficient parallel code without requiring much interaction with the programmer.

\section{The contributions of this paper are:}

- We abstract a wide variety of stencil computations into a set of domain-specific specifications. This allows the end-user to customize specific problems without having to consider the underlying architecture.

- We thoroughly summarize optimization techniques for stencil problems in previous literature and extract three sets of key parameters that affect the performance: (1) Block sizes that determine the shared-memory usage per block; (2) block dimensions that affect the number of registers consumed by each thread and (3) whether or not to map a subset of the input into texture memory.

- We develop an auto-generation and auto-tuning framework, i.e., we translate stencil specifications into executable code that is subsequently auto-tuned to the optimal configuration within a parametrized search space for each target GPU.

- Experimental results show competitive performance to manual tuning and demonstrate the superiority and necessity for autotuning to combining performance with correctness.

The rest of the paper is organized as follows. The related work is presented in Section 2. In Section 3, we describe the stencil specification and the output of the framework. We explain various optimization strategies and how they are applied to our framework in Section 4. Detailed experimental results are presented in Section 5 , with thorough comparison with previous works. We summarize our work in Section 6.

\section{RELATED WORK}

Auto-tuning has long been identified as an effective approach to offer portability and productivity. For example, ATLAS [2], OSKI [23] and FFTW [6] are well recognized auto-tuning libraries targeted at general-purpose processors for dense/sparse linear algebra subroutines and FFT kernels in digital signal processing, respectively.
Recent improvements in programmability of GPUs allow autotuning to be applied to GPUs as well. Several CUDA implementations for linear algebra subroutines and FFTs with auto-tuning capability already exist $[7,12,19]$.

Previous implementations of stencil computations on GPUs can be grouped into three categories: (1) Hand-coded implementations of a particular stencil that strive to achieve the best performance possible $[17,18,20]$ - but with optimization techniques that may not generalize to other types of stencils - (2) Implementations where ease of programming is the primary goal rather than performance - often with code generators for various stencils $[5,22,14,11]$ and (3) implementations that focus on a particular parameter and study how tuning it can affect performance $[13,16]$.

We conjecture that performance or programmability are not mutually exclusive. The merit of our work is to offer both ease of programming and performance at the same time. By providing a stencil specification front-end, we alleviate the end-user's burden to master architectural details. Near-optimal performance is achieved by extracting necessary parameters and thoroughly autotuning them. Even though some of the aforementioned work utilizes certain tuning parameters, such work either relies on ad-hoc hand tuning [22] or the tuning space is limited [5, 11].

\section{DESIGN OVERVIEW}

The stencil computation considered in this work allows point-wise updates according to a sequence of the following equation over a $3 \mathrm{D}$ rectangular domain:

$$
\begin{aligned}
\operatorname{out}([i][j][k]) & =\sum_{m} w_{m} * i n\left[i \pm I_{m}\right]\left[j \pm J_{m}\right]\left[k \pm K_{m}\right] \\
& +\sum_{l} w_{l}[i][j][k] * i n\left[i \pm I_{l}\right]\left[j \pm J_{l}\right]\left[k \pm K_{l}\right] \\
& +\sum_{n} w_{n} * i n_{n}
\end{aligned}
$$

The three dimensional addressing in the parenthesis on the left hand side is optional. If absent, we assume the result (out in this case) is an intermediate result that will be used later in another instruction on the right hand side as an input $i n_{n}$. The first two parts on the right hand side characterize the stencil behavior. The center point and a number of neighboring points in the input grid (in) are weighted by either scalar constants $\left(w_{m}\right)$ or elements in grid variables $\left(w_{l}[i][j][k]\right)$ at the same location as the output. Offsets $\left(I_{m} / J_{m} / K_{m}\right.$ and $\left.I_{l} / J_{l} / K_{l}\right)$ that constrain how the input grid is accessed are all constant. We call their maxima the halo margins of three dimensions (halo_ $i=\max \left\{I_{m / l}\right\}$, halo_ $j=\max \left\{J_{m / l}\right\}$ and halo_k $\left.=\max \left\{K_{m / l}\right\}\right)$. To ensure that the access pattern is legal (non-negative indexing) for marginal elements in the input grid $i n$, we assume both input and output grids (in and out) are enlarged by twice the halo margins on each associated dimension.

We differentiate $w_{l} \mathrm{~s}$ and $i n$ in (1) and call them array parameters and array input, respectively. Array parameters are restricted by their access pattern: they can only be accessed at the same position as the output element. The array input can be accessed with various constant offsets $(i / j / k \mathrm{~s})$ on each dimension. We assume there is only one array input, but there can be zero or multiple array parameters.

Given the stencil specification that contains only a list of instructions in the format of Eq. 1, our auto-tuning framework generates 
typedef struct \{

int dims[3];

int iter;

int haloMargins[2][3];

int numNodes; // for multi-node

int curNode;// for multi-node

\} StencilConfig;

(a) Auto-Generated Code

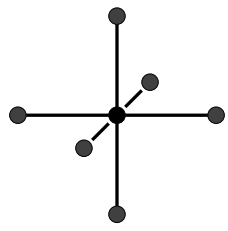

(a) 7-Point
initStencil(StencilConfig * config); stencilIteration(StencilConfig *); stencilIteration_mpi(StencilConfig *); exitStencil(StencilConfig *);

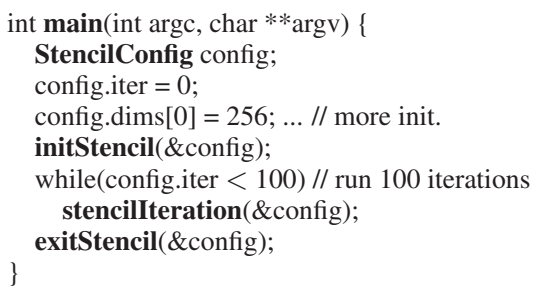

(c) Sample User Code

Figure 1: Example of Auto-Generated Code (Excerpts)

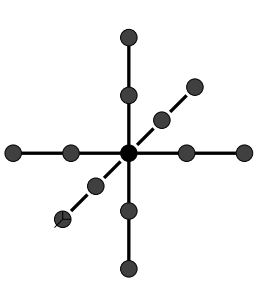

(b) 13-Point

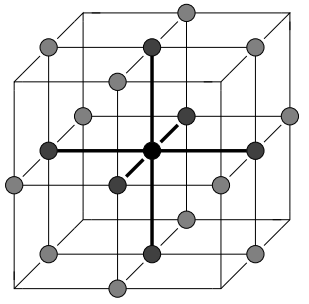

(c) 19-Point

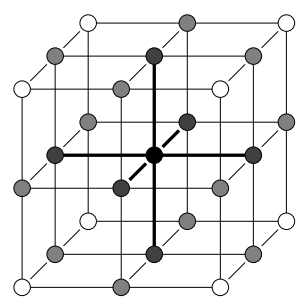

(d) 27-Point

Figure 2: Stencil Examples

a header file and an implementation file that can be either included in user code or compiled into libraries.

Excerpts of the generated code are depicted in Figure 1. The two major APIs are stencilIteration() and stencillteration_mpi(). One performs single GPU calculations, the other is for multiple-node GPUs (GPU clusters) computations with node-to-node MPI message passing.

We call a stencil calculation an $\mathrm{N}$-point stencil where $\mathrm{N}$ is the total number of input points used to calculate one output point and an order-M stencil where $\mathbf{M}$ is the maximum over all halo_ $i / j / k \mathrm{~s}$. In this paper, we choose four types of stencil computations as benchmarks (see Figure 2).

- 7-Point Stencil (Figure 2(a)): Each element in the output grid is updated by the same position in the input grid and 6 neighbors offset by 1 on each direction. The grid point and 6 neighbors are scaled by $\alpha$ and $\beta$, respectively, before they are added to generate the output. Both $\alpha$ and $\beta$ are constants. There are 8 floating-point operations for each point ( 6 adds and 2 multiplies).

- 13-Point Stencil (Figure 2(b)): The access pattern resembles the 7-point stencil except that the maximal distance to the neighbors extends to 2, making it an order-2 stencil. There are 15 floatingpoint operations at each point (12 adds and 3 multiplies).

- 19-Point Stencil (Figure 2(c)): This is also called the Himeno benchmark, the behavior of which is detailed elsewhere [20]. We use the same specification (Table I in [20]), except for ignoring the last line of residual calculation. All the weights in this benchmark are array parameters, making it a very cache-unfriendly benchmark. The total number of floating-point operations is 32 and there are 14 memory accesses per point.

- 27-Point Stencil (Figure 2(d)): Each grid point computation involves all points in a $3 \times 3 \times 3$ cube surrounding the center grid point. The 4 edge points, 8 corner points and 12 face neighbor points are multiplied by different constants. The number of operations is 30 with 4 multiplies and 26 adds.

Table 1 summaries the specifications and properties of the four stencils above.

\subsection{Domain Specification and Framework}

The formulation of a stencil is trivial in our framework as users provides a file specifying an equation according to the format of Eq. 1 plus parameters, such as the size of each dimension and data type (float or double). Table 1 shows that each stencil can be expressed by no more than a few lines of code. In contrast to hand-written CUDA kernels, which usually are a hundreds of lines of code, this is a considerable improvement in terms of productivity. The internal work flow of the framework is depicted in Figure 3. The parser analyzes the specification code in terms of Eq. 1 and extracts stencil features. These include halo margins (halo_ $i / j / k$ ), input/output array names, scalar or array parameters $(w s)$ and the number of floating-point operations per stencil. The parser also detects whether the stencil access pattern includes corner element accesses or not. 7-point and 13-point stencils are corner access free because at most one dimensional offset exists when accessing the input array. The code generator takes those feature parameters and chooses different template files according to the corner access pattern before generating tunable code. The auto-tuning engine mainly operates on a single-node level, where optimized parameters are determined based on run-time profiling. The same optimized parameters are used on multiple nodes to generate GPU cluster code with MPI support.

\subsection{Domain Kernel Template}

The design of the template kernel file is affected by the strategy to break the $3 \mathrm{D}$ rectangular space into thread blocks in CUDA. In related work, the $3 \mathrm{D} X \times Y \times Z$ space was divided into smaller cuboids of size $x \times y \times z[5,15]$. Each of them was mapped to a thread block of the same size. Recently, a 2.5D decomposition method was proposed [20, 18]. It decomposes the 3D stencil space over the two most frequently changed dimensions (X and Y). Sten- 


\begin{tabular}{|c|c|c|c|c|}
\hline Kernel & Specification & $\begin{array}{l}\text { \# array } \\
\text { params }\end{array}$ & $\begin{array}{l}\text { Flops } \\
\text { per stencil }\end{array}$ & $\begin{array}{l}\text { mem. refs } \\
\text { per stencil }\end{array}$ \\
\hline \begin{tabular}{c|}
7 -point \\
order-1
\end{tabular} & $\begin{array}{l}t m p=\left(u_{i+1, j, k}+u_{i-1, j, k}+u_{i, j+1, k}+u_{i, j-1, k}+u_{i, j, k+1}+u_{i, j, k-1}\right) * \text { beta; } \\
u 1_{i, j, k}=\text { tmp }+ \text { alpha } * u_{i, j, k}\end{array}$ & 0 & 8 & 8 \\
\hline $\begin{array}{l}\text { 13-point } \\
\text { order-2 }\end{array}$ & $\begin{array}{l}\operatorname{tmp}=\text { coef } 1 *\left(u_{i+1, j, k}+u_{i-1, j, k}+u_{i, j+1, k}+u_{i, j-1, k}+u_{i, j, k+1}+u_{i, j, k-1}\right) \\
\operatorname{tmp}+=\text { coef } 2 *\left(u_{i+2, j, k}+u_{i-2, j, k}+u_{i, j+2, k}+u_{i, j-2, k}+u_{i, j, k+2}+u_{i, j, k-2}\right) \\
u 1_{i, j, k}=\text { tmp }+ \text { coef } 0 * u_{i, j, k}\end{array}$ & 0 & 15 & 14 \\
\hline $\begin{array}{l}\text { 19-point } \\
\text { order-1 } \\
\text { (himeno) }\end{array}$ & $\begin{array}{l}s 0=w r k 1_{i, j, k}+a 0 d_{i, j, k} * p_{i, j, k+1}+a 1 d_{i, j, k} * p_{i, j+1, k+1} \\
s 0+=b 0 d_{i, j, k} *\left(p_{i, j+1, k+1}-p_{i, j-1, k+1}-p_{i, j+1, k-1}+p_{i, j-1, k-1}\right)+a 2 d_{i, j, k} * p_{i+1, j, k} \\
s 0+=b 1 d_{i, j, k} *\left(p_{i+1, j+1, k}-p_{i-1, j+1, k}-p_{i+1, j-1, k}+p_{i-1, j-1, k}\right) ; \\
s 0+=b 2 d_{i, j, k} *\left(p_{i+1, j, k+1}-p_{i-1, j, k+1}-p_{i+1, j, k-1}+p_{i-1, j, k-1}\right)+c 0 d_{i, j, k} * p_{i, j, k-1} ; \\
s 0+=c 1 d_{i, j, k} * p_{i, j-1, k}+c 2 d_{i, j, k} * p_{i-1, j, k} \\
s s=\left(s 0 * a 3 d_{i, j, k}-p_{i, j, k}\right) * b n d_{i, j, k} ; \\
w r k 2_{i, j, k}=p_{i, j, k}+\text { omega } * s s ;\end{array}$ & 12 & 32 & 32 \\
\hline $\begin{array}{c}27 \text {-point } \\
\text { order-1 }\end{array}$ & $\begin{array}{l}b_{i, j, k}=\text { param0 } * a_{i, j, k} \\
+ \text { param } 1 *\left(a_{i-1, j, k}+a_{i+1, j, k}+a_{i, j-1, k}+a_{i, j+1, k}+a_{i, j, k-1}+a_{i, j, k+1}\right) \\
+ \text { param } 2 *\left(a_{i-1, j-1, k}+a_{i-1, j+1, k}+a_{i+1, j-1, k}+a_{i+1, j+1, k}+a_{i-1, j, k-1}+a_{i-1, j, k+1}\right. \\
\left.+a_{i+1, j, k-1}+a_{i+1, j, k+1}+a_{i, j-1, k-1}+a_{i, j-1, k+1}+a_{i, j+1, k-1}+a_{i, j+1, k+1}\right) \\
+ \text { param } *\left(a_{i-1, j-1, k-1}+a_{i-1, j-1, k+1}+a_{i-1, j+1, k-1}+a_{i-1, j+1, k+1}\right. \\
\left.+a_{i+1, j-1, k-1}+a_{i+1, j-1, k+1}+a_{i+1, j+1, k-1}+a_{i+1, j+1, k+1}\right)\end{array}$ & 0 & 30 & 28 \\
\hline
\end{tabular}

Table 1: Specifications of Four Stencil Benchmarks. Indices are subscripted to save space.

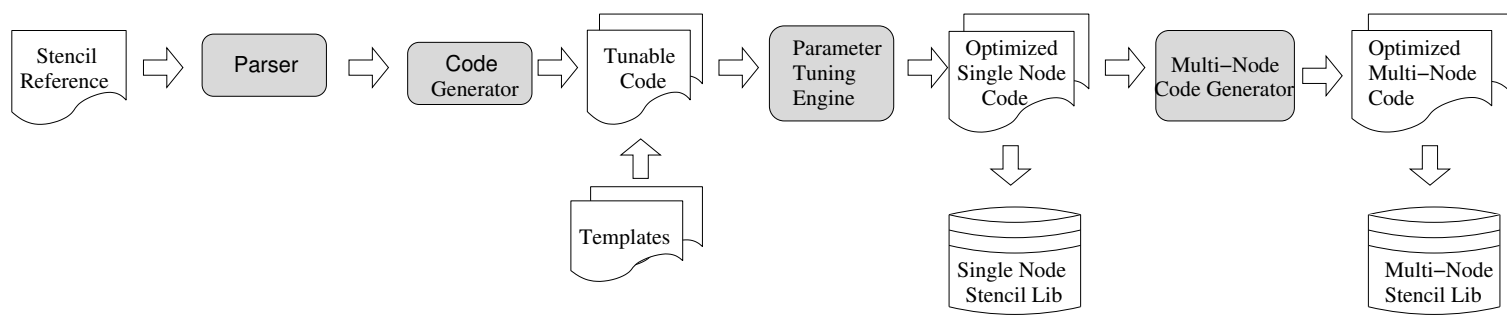

Figure 3: System work flow: A user-defined specification is parsed to generate tunable code based on a template. The code is passed to an auto-tuning system to find the best parameter configuration for a single GPU (also for GPU clusters with MPI)

cils of size $x \times y \times Z$ are assigned to a thread block, which contains only a plane of $x \times y$ threads. Inside the kernel, threads sweep over the $\mathrm{Z}$ axis and cooperatively process one plane at a time.

The benefits of the second method are three-fold: (1) It reduces the pressure on shared memory usage. In 3D decomposition, each block maintains a small block of size $\left(x+2 \times\right.$ halo $\left.\_i\right) \times(y+2 \times$ halo_j $) \times(z+2 \times$ halo_k $)$ in shared memory. The $2.5 \mathrm{D}$ method only needs a blocks size of $(x+2 \times$ halo_ $i) \times(y+2 \times$ halo_ $j) \times$ $\left(1+2 \times h a l o \_k\right)$. While sweeping through the z-axis, the planes can be shifted and reused as the work on $\mathrm{z}$-axis is progressed. If the stencil does not have corner accesses, such as 7-point and 13point stencils, we can further reduce the shared-memory usage to $(x+2 \times$ halo_ $i) \times(y+2 \times$ halo_ $j)$ while keeping the other parameters in registers. (2) The $3 \mathrm{D}$ decomposition method consumes more memory bandwidth on the $\mathrm{Z}$ axis because halo regions on $\mathrm{Z}$ are loaded twice on different blocks along the $\mathrm{Z}$ axis. (3) The $2.5 \mathrm{D}$ decomposition method tends to allocate more stencil points per thread ( $\mathrm{Z}$ points per thread instead of $\mathrm{z}$ points). This is an optimization technique also known as thread fusion. For a large enough problem size, i.e., $(X \times Y)$ generates enough threads, this helps to amortize other overheads, such as initial setup code in the kernel.

In our design, we adopt the block partition strategy in the $2.5 \mathrm{D}$ blocking method, i.e., stencil space is partitioned into columns (Figure 4(a)). The cross section of each column is of size (BlockSize.x $\times$ BlockSize.y), see Figure 4(b). We further unroll over both $\mathrm{X}$ and $\mathrm{Y}$ dimensions to use (BlockDim. $\times$ BlockDim.y) threads per kernel block (see Figure 4(c)). Previous work only exploits the unrolling factor at most over the Y dimen- sion. Our experiments illustrate that unrolling over both dimensions can be beneficial (see Section 5).

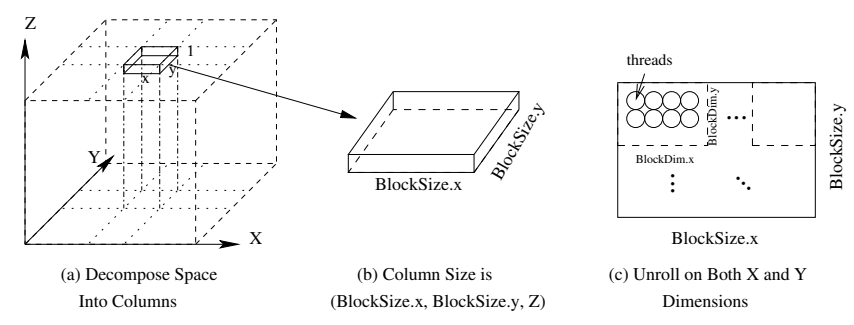

Figure 4: Stencil space decomposed over X \& Y; process one column per thread block; thread code is unrolled.

Our code generator is based on two kernel templates, depending on whether the stencil has corner accesses (Fig. 5(a)) or not (Fig. $5(\mathrm{~b})$ ), where halo_k $=1$ is assumed in these figures. Their most distinct difference is how the shared memory is used. For stencils with corner accesses, all input stencils are first stored in shared memory to calculate the output stencils. The corner-free stencils can be treated as a special case where a plane of stencils does not share inputs other than the points on the same plane. Therefore, only the middle plane is stored in shared memory in this case all other inputs along the $\mathrm{Z}$ axis are stored in register files. This approach, tailored to corner-free stencils, not only reduces the shared memory pressure but also speeds up the stencil calculation due to the performance advantage of using registers over shared memory. 


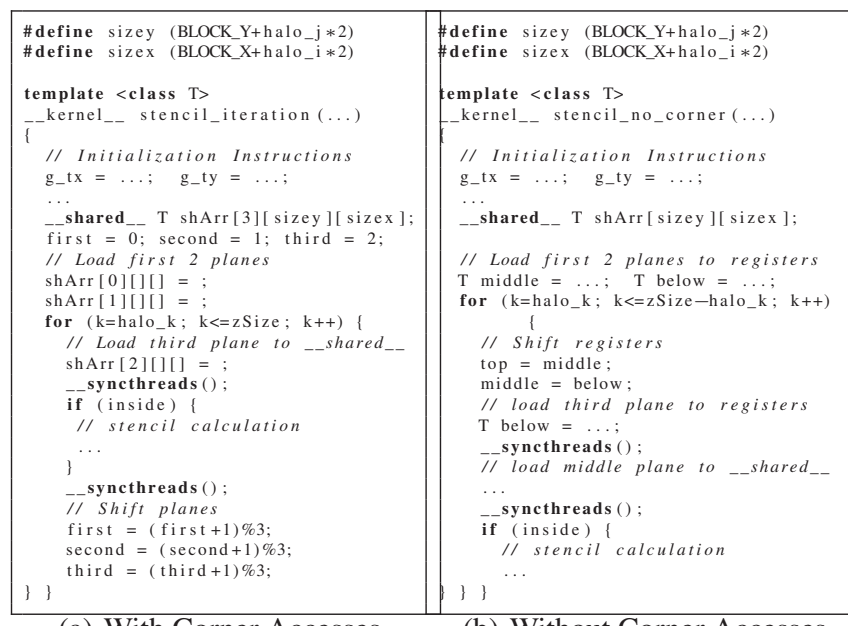

(a) With Corner Accesses

(b) Without Corner Accesses
Figure 5: Stencil Kernel Templates

\section{GPU-SPECIFIC AUTO-TUNING}

We next describe in detail various optimization techniques used by our implementation. We reason about their effects on performance and consider if they need to be made elastic by promoting them as parameters for auto-tuning.

\subsection{Single Node Optimizations}

Coalescing Memory Accesses: For NVIDIA GPUs, the latency of global memory references is deeply affected by whether the memory is accessed in coalesced way or not. More recent GPUs support coalesced memory access when memory accesses conducted by threads in one warp can be combined into as few memory transactions as possible [1], where a warp is the basic thread instruction scheduling unit in NVIDIA GPUs. We reinforce the following rules to coalesce most of the memory accesses:

- The size of the most frequently changing dimension ( $\mathrm{X}$ dimension) for input/output arrays is padded to multiples of 32 stencil elements.

- The origin of the input/output arrays are shifted right by $32-$ $H A L O \_I$ stencil elements relative to the memory pointer obtained from the CUDA malloc function. This guarantees 128-bit alignment. The internal origins of the input/output array thus become 128-bit aligned ensuring coalesced memory accesses for output arrays as long as every thread loads the same row at the same time when operating on a half-warp granularity.

- Parameter arrays are allocated to be the same size as the input/output array, even though only the internal elements are used throughout the stencil calculation. This way, the indices of parameter arrays and parameter input become identical saving registers and extra cycles for address calculations. Similar to the input/output arrays, their origins are also shifted to the right. Reading from the parameter arrays become coalesced as well.

Tuning the Block Size: Choosing the right block size is one of the most important factors to balance the utilization of registers and shared memory. Since we use Z-axis sweeps, our blocks have two dimensions of size BlockSize. $x \times$ BlockSize.y. The optimal blocking size is determined by several seemingly conflicting factors:
- Since accesses to part of the halo margins are non-coalesced memory accesses, we want to limit these as much as possible. This gives us incentive to increase BlockSize. $x$ as much as possible.

- To reduce the redundant loading of halo margins between different blocks, we need to keep the block close to a square shape.

- The shared-memory usage is proportional to BlockSize. $\times$ BlockSize.y. It must not surpass the shared-memory size on-chip.

Our experiments show that the optimal blocking size can be different under different scenarios: On one hand, different GPU models require different sizes for the same stencil problem. On the other hand, the same GPU model requires different blocking sizes for different stencil problems. To obtain the coalesced memory access effects for an input array, our search space for BlockSize.x is a multiple of the half-warp $(16,32,48,64)$. BlockSize.y has no such constraints. So we sweep its value continuously from 2 to 16. The search space for the CUDA block size (BlockDim.x and BlockDim.y) is a subset of the block size search space, with the constraint that BlockSize.x/y is integer divisible by BlockDim.x/y. The motivation behind this ratio is that a smaller set of threads has a higher efficiency in using registers. This thorough search lets us balance register utilization and shared memory space, two key resources for stencil implementations on GPUs that are scarce.

Loading the Input Array Efficiently: An important step in the stencil kernel is to efficiently access the input array. A straightforward but naive implementation is to load it directly from the off-chip global memory while calculating the output point. The obvious drawback is that this does not exploit the data sharing between neighboring threads. The on-chip shared memory serves as an ideal user-controlled scratch pad in this scenario. The problem narrows down to how to efficiently load a larger block of data $\left(\left(\right.\right.$ BlockSize. $\left.x+2 * H A L O \_I\right) \times($ BlockSize.y + $\left.2 * H A L O \_J\right)$ ) using a smaller set of computation threads (BlockDim.x $\times$ BlockDim.y). We first load the internal region (BlockSize.x $\times$ BlockSize.y). Because BlockDim.x/y are divisible by BlockSize.x/y, this can be done easily without branches. For marginal regions, we rely on the code generator to map computational threads to elements on the margin region, as shown in Figure 6. In the graph, we assume BlockDim.x/y equals to BlockSize.x/y, respectively. Each computing thread is sequentially assigned to a point in the margin area. The $\mathrm{x}$ and $\mathrm{y}$ indices are auto generated as a constant array. The number of points in the margin area is not necessarily divisible by the number of computing threads. In those cases, threads will be responsible for loading more than one marginal points or there will be idle threads that load the upper-left corner point (see the Figure 6(a)) to avoid diverging branches. Comparing with other approaches, e.g., [20], this method neither requires branches nor issues any unnecessary loads. The only non-coalesced memory loads are issued for the columns on each side of the sub-plane.

Using Texture Memory: Mapping the read-only input array into the GPU's texture memory has been shown to improve performance in [20], especially for bandwidth-limited benchmarks. There is no texture support for the double precision data type, but we can use the texture fetch for the int 2 type and _ hiloint2double to convert it to double. Whether or not to use texture memory for the input array is determined by a boolean tuning parameter. 


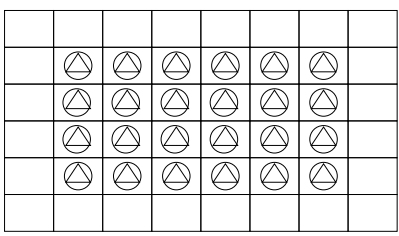

(1)

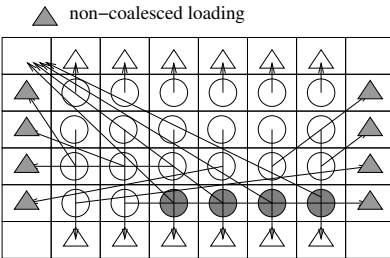

(2) idle thread

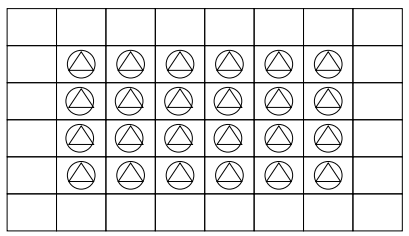

(1)

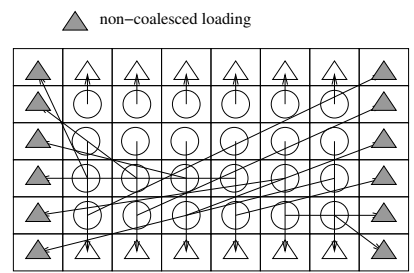

(2)

(a) No Corner Case

Figure 6: Load input sub-plane to shared memory. Internal regions are loaded in Step (1). There is a one-to-one mapping between computing threads and internal regions. In Step (2), the mapping is auto-generated by the parameter tuning engine. (A circle denotes a thread. A triangle denotes an array element loaded at the current step.)

\subsection{Multi-Node Auto-Tuning}

For GPU clusters, we divide the stencil space along the Cartesian space. Each node is responsible for updating a smaller rectangular $3 \mathrm{D}$ space. The tuning parameters determined for a single node are re-used directly for multi-node scenarios. However, the code generator needs to break the single kernel into several smaller ones, each of which only processes a portion of the data set. The objective is to separate the six plane boundaries from the internal region. While the boundaries need to be exchanged between neighboring nodes, the internal regions can be calculated completely in parallel with communication.

Our framework generates MPI calls for inter-node communication. Nodes perform the following steps per iteration:

(1) Kernels copy non-continuous boundaries residing in GPU memory into continuous GPU memory buffers. For stencils with corner accesses, eight corners and 12 edges are also copied into separate buffers. Then, continuous boundaries are transferred from GPU memory to host memory via cudaMemcpy.

(2) An asynchronous kernel updates internal regions.

(3) MPI sends and receives are issued to exchange boundaries. Once boundaries are received, boundaries are copied from host memory to GPU memory. This step can be overlapped with the step (2).

(4) Kernels update stencils on boundaries.

These steps are illustrated in Figure 7.

\section{EXPERIMENTAL RESULTS}

\subsection{Experimental Setup}

We conducted experiments on single nodes with four NVIDIA GPU models: Geforce GTX 280, Tesla C1060, Tesla C2050 and Geforce GTX 480, spanning two generations of NVIDIA GPUs ranging from consumer-end graphics card to high performance computing GPUs. Their major specifications are listed in Table 2. All kernels are compiled under CUDA 3.2 at $\mathrm{O} 3$ optimization level. Experiments with Tesla C2050 are conducted with ECC turned off. For Fermi GPUs (Tesla C2050 and GTX 480), we prefer shared memory over L1 cache since the shared memory size is $48 \mathrm{~KB}$ (in contrast to $16 \mathrm{~KB}$ in earlier GPUs).

We also conducted multi-node experiments on two set of GPU clusters connected by QDR Infiniband (36 Gbps) with fat-tree topology.

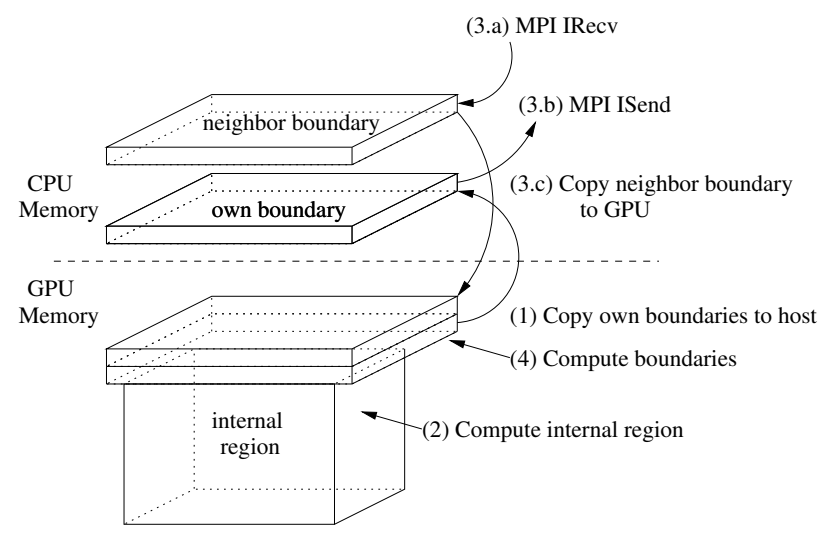

Figure 7: Steps in multi-node scenario. For clarity, only one boundary plane is shown.

One cluster was comprised of 32 nodes, each with one Tesla C2050, other had 48 nodes, each with one GTX 480.

\subsection{Single Node Results}

Our single-node auto-tuning engine finds the optimal parameters for all stencil types on each GPU model within the given search space. These parameters are shown in Table 3. Each GPU model has different optimal settings for all stencil types, even within the same GPU generation. Almost all models favor large BlockSize.x except for some cases with early generation GPUs. These older GPUs have tighter restrictions on shared memory size, especially for double precision (DP) stencils. Thus, they can only afford smaller BlockSize.x sizes. BlockSize.y is usually less than BlockSize.x, except for 7/13-point DP stencils on a GTX 280 and the 13-point DP stencil on a Tesla C1060 because their smaller BlockSize.x (16) allows them to have a larger BlockSize.y. Thus, reducing the non-coalesced memory access (increasing BlockSize.x) is favored over reducing redundant loads (increasing BlockSize.y).

An illustration of each tuning parameter's contribution to performance is given in Figure 8. Here, auto-tuning is comprised of three steps: (1) BlockSize.x/y are set to be equal to BlockDim.x/y; (2) BlockSizes.x/y are tuned for better performance; (3) texture mapping is enabled/disabled. The necessity to unroll is confirmed by the fact that BlockDim.x/y sizes are almost always different than BlockSize.x/y. The only exception is given by a 19-point DP stencil for Fermi GPUs. In this cases, BlockSize.y is too small 


\begin{tabular}{|c|c|c|c|c|c|c|c|c|}
\hline Model & SM Count & Core Count & L1 Cache & Bandwidth(GB/s) & Register File Size & Shared Memory & SP GFlops & DP GFlops \\
\hline \hline Geforce GTX 280 & 30 & 240 & $\mathrm{~N}$ & 141.7 & $16 \mathrm{~KB}$ & $16 \mathrm{~KB}$ & 933 & 78 \\
\hline Tesla C1060 & 30 & 240 & $\mathrm{~N}$ & 102.4 & $16 \mathrm{~KB}$ & $16 \mathrm{~KB}$ & 933 & 78 \\
\hline Tesla C2050 & 14 & 448 & $\mathrm{Y}$ & 144 & $32 \mathrm{~KB}$ & 16 or 48 KB & 1288 & 515 \\
\hline Geforce GTX 480 & 15 & 480 & $\mathrm{Y}$ & 177.4 & $32 \mathrm{~KB}$ & 16 or 48 KB & 1345 & 168 \\
\hline
\end{tabular}

Table 2: Single Node Experiment Platforms

\begin{tabular}{|c|c|c|c|c|c|c|}
\hline Model & BlockSize.x & BlockSize.y & BlockDim.x & BlockDim.y & Texture & SP GFlops \\
\hline \hline Geforce GTX 280 & $64 / 32 / 64 / 16$ & $8 / 8 / 3 / 6$ & $32 / 32 / 64 / 16$ & $8 / 2 / 3 / 2$ & Y/Y/N/N & $76.0 / 117.0 / 57.6 / 94.2$ \\
\hline Tesla C1060 & $64 / 64 / 64 / 32$ & $8 / 6 / 6 / 8$ & $32 / 64 / 64 / 32$ & $8 / 2 / 3 / 2$ & Y/N/Y/N & $57.5 / 91.8 / 44.8 / 95.5$ \\
\hline Tesla C2050 & $64 / 64 / 64 / 64$ & $8 / 6 / 3 / 4$ & $32 / 64 / 32 / 32$ & $8 / 3 / 3 / 4$ & Y/Y/N/Y & $87.3 / 133.8 / 64.6 / 157.6$ \\
\hline Geforce GTX 480 & $64 / 64 / 64 / 64$ & $3 / 3 / 3 / 8$ & $32 / 32 / 32 / 32$ & $3 / 3 / 3 / 4$ & Y/Y/N/Y & $108.2 / 167.8 / 77.4 / 203.7$ \\
\hline \hline Model & BlockSize.x & BlockSize.y & BlockDim.x & BlockDim.y & Texture & DP GFlops \\
\hline \hline Geforce GTX 280 & $16 / 16 / 16 / 16$ & $16 / 16 / 6 / 6$ & $16 / 16 / 16 / 16$ & $4 / 8 / 3 / 3$ & N/N/Y/N & $32.5 / 35.4 / 24.0 / 29.0$ \\
\hline Tesla C1060 & $32 / 16 / 32 / 16$ & $6 / 16 / 4 / 6$ & $32 / 16 / 32 / 16$ & $2 / 8 / 2 / 3$ & N/N/Y/N & $28.8 / 35.3 / 22.8 / 29.3$ \\
\hline Tesla C2050 & $64 / 32 / 64 / 32$ & $8 / 6 / 3 / 6$ & $32 / 32 / 64 / 32$ & $4 / 2 / 3 / 2$ & Y/Y/N/Y & $45.9 / 66.8 / 31.8 / 97.7$ \\
\hline Geforce GTX 480 & $64 / 32 / 64 / 32$ & $6 / 6 / 3 / 4$ & $32 / 32 / 64 / 16$ & $3 / 2 / 3 / 4$ & Y/Y/N/Y & $55.2 / 77.2 / 38.7 / 86.0$ \\
\hline
\end{tabular}

Table 3: 7/13/19/27-Point Stencil Optimal Tuning Results on Single GPU for Single/Double Precision (SP/DP)

to unroll. In addition, Fermi GPUs provide enough registers to support a BlockDim.x of the same size as BlockSize.x.

Another interesting observation is that mapping the input array to texture memory does not necessarily result in better performance. This is in part because some stencils are not bandwidth-limited on certain GPUs. For GPUs that have high GFlops capabilities, using texture memory usually helps because memory references are on the critical path (7/13/27-point DP stencils for C2050 and GTX 480). Using texture memory has one overhead though: Texture mapping requires the device memory to start from 128-bit aligned address. But our input/output array base addresses are shifted to non-aligned addresses so that the addresses with offset at halo_i (base address for internal region) are 128-bit aligned. Therefore, there is an extra offset adjustment calculation if we want to enable texture mapping. This extra arithmetic for address computation can negate the benefit of lower latencies for texture memory accesses for some cases.

To demonstrate the effectiveness of the auto-tuning engine, we select two cases and represent performance in GFlops as a surface in a 3D histogram. Figure 9 depicts the single-precision (SP) 7-point stencil on a GTX 280. Figure 10 depicts the DP 27-point stencil on a Tesla C2050. The left diagrams in the figures illustrate how the performance changes while varying BlockSize.x/y, assuming the best BlockDim.x/y has been found. The right diagrams in the figures depicts how the performance changes when varying BlockDim.x/y for a fixed BlockSize.x/y overall. The figures demonstrate that each tuning parameter plays an important role in the final performance, neither one of which can be explored independently of the other. Hence, an auto-tuner needs to exhaustively test all permutations.

Our auto-tuning engine does exactly that: an exhaustive search over all possible permutations is performed. This guarantees a global optimum with respect to the parameter search space. Adaptive search methods could be adopted to prune the search space. However, care must be taken because local optima exist, as seen in the figures. For example, in Figure 9(b), $(64,4)$ is another locally optimal BlockDim.x/y pair. Considering the search space is rela- tively small (less than 200 combinations in the worst case), exhaustive search is feasible as individual runs can be short.

\subsection{Multi-Node Results}

We study the weak scaling property [8] of our framework in the two GPU clusters. We keep the problem size per GPU constant and increase the stencil size over all three dimensions at roughly the same rate as the increase in number of GPUs. Therefore, the stencil space is kept as close to a cube as possible. The $\mathrm{Y}$ axis of Figure 11 depicts the normalized performance (measured in GFlops) of a single GPU. For the C2050 GPU cluster, all three order-1 stencils (7/19/27-point) show better efficiency (77\% to $80 \%$ ) than order-2 stencil (50\% for 13-point). Because the GTX 480 has higher singlenode DP GFlops for 7/13/19-point stencils, the weak scaling efficiency is worse than that on the $\mathrm{C} 2050$ cluster. But for 27-point stencils, GTX 480's single-node DP GFlops is less than C2050's DP GFlops. Therefore, the efficiency is better (about 90\%). This can be explained by the difference in inter-node message sizes required by different stencils types. The message size is roughly proportional to the degree of the stencil order. Therefore, our 13-point stencil is communication-bound in our current cluster configuration.

Some of the curves do not show a noticeable improvement from 24 to 27 GPUs (nodes). The 19-point stencil curve even shows a slight drop. This is because the stencil space is divided into $2 \times 3 \times 4$ and $3 \times 3 \times 3$ partitions in these two cases, respectively. The latter case contains a center node that needs to communicate with all other 26 nodes. This node becomes a hot-spot and reduces the performance. But as we increase the number of GPUs, the curve recovers to the expected slope for weak scaling.

\subsection{Comparison with Previous Work}

We report our results on a wide range of GPUs and stencil types, which allows us to compare our performance directly with a wide range of prior work, both for handwritten and auto-generated codes.

Datta et al.'s work on optimizing stencil codes in multi-core architectures including GPUs is one of the early contributions in this area [5]. They showed an unprecedented 36 GFlops for 7-point 


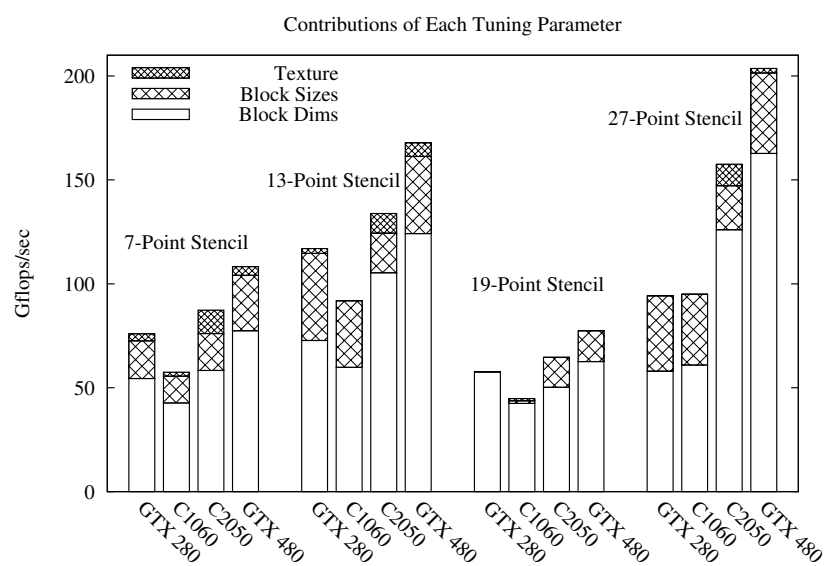

(a) Single Precision (SP) Stencils

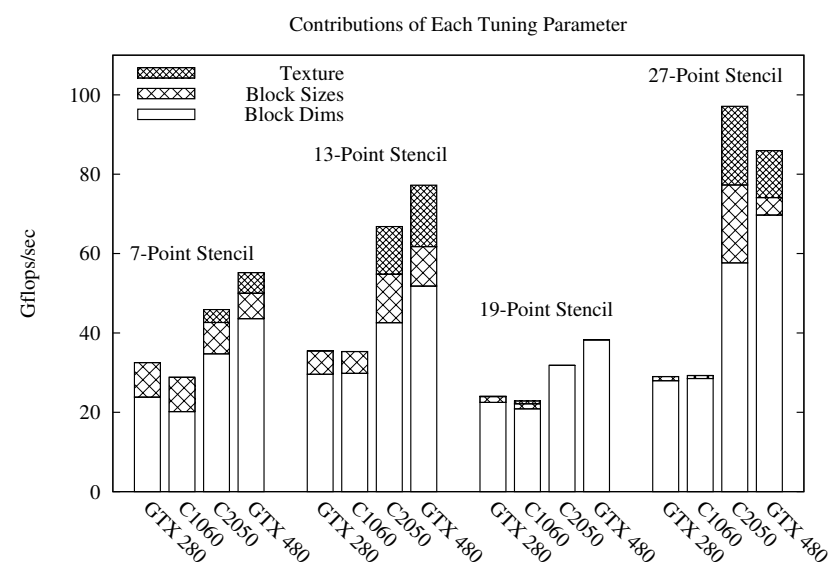

(b) Double Precision (DP) Stencils

Figure 8: Stencil Tuning Effect Breakups

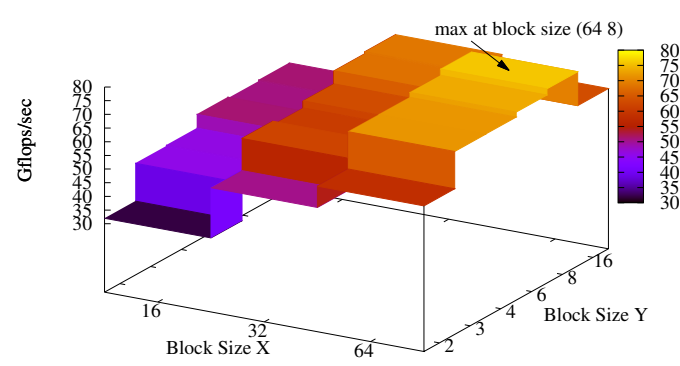

(a) Find Optimal Block Sizes

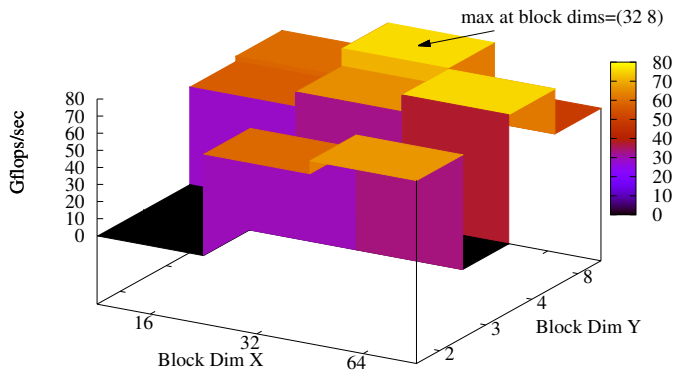

(b) Find Optimal Block Dims, Fixed Block Size at (64,

Figure 9: GTX 280 7-Point Stencil (SP)

stencil on a GTX 280 with their highly optimized code. Theirs is $10 \%$ faster than our performance (32.5 GFlops). This is mainly due to the difference between the instruction orders in our template file and their hand-tuned kernel code, as we discovered by inspecting their and our codes side-by-side. But interestingly, their best performance is achieved at a block size of $16 \times 16$ and unroll factor of 4 over the dimension $\mathrm{Y}$, which is consistent to our findings in our auto-tuning engine. However, this configuration is only optimal for a DP 7-point stencil on the GTX 280s. For everything else, the $16 \times 16$ block sizes are no longer optimal, as indicated by Table 3 .

An efficient and handwritten CUDA implementation on the Himeno benchmark is reported by Philips et al. [20]. Their implementation, with an extra two Flops per stencil for residual calculation, achieved 50 GFlops SP on a Tesla C1060. Our auto-generated code achieves 44.8 GFlops on the same platform and is within $5 \%$ to theirs if Flops are normalized $\left(44.8 \times \frac{34}{32}=47.6\right)$. Their best block sizes are $64 \times 2$ for Tesla C1060, while ours is $64 \times 6$ with an unrolling factor of 2 over the $\mathrm{Y}$ axis. This is because they load the input arrays into shared memory by issuing four branch-free loads aligned at four corners. Choosing BlockSize.y as 2, in their case, minimizes redundant memory loads, which is beneficial because SP Himeno is bandwidth limited on the $\mathrm{C} 1060$. They also reported near-perfect weak scaling efficiency on up to 16 GPUs. But their system configuration is different from ours: (1) Each node has two GPUs instead of one in our case. Therefore, half of the network messages become memory copies on the same host. (2) The stencil space only grows along the $\mathrm{Z}$ axis, eliminating the need to perform Cartesian partitioning. This reduces the multi-node code complexity significantly.

Kamil et al. proposed an auto-tuning framework for multi-core architectures [11]. However, they reported only 14 GFlops DP on a 7-point stencil for a GTX 280. This is mainly because their code generator does not take advantage of the fast on-chip shared memory, which is an ideal intermediate storage level to reduce memory load for stencil-like computations.

Nguyen et al. have reported by far the fastest implementation of any SP stencil code on single GPU [18]. Their manually-written code for a 7-point stencil achieves 136 GFlops on GTX 285 (a similar platform as GTX 280), a large gain over our reported 76 GFlops. However, their extra speedup comes from saving a large amount of global memory accesses by exploiting data locality on the time domain. This is equivalent to executing several iterations per kernel, a technique also known as increasing the ghost region. Increasing the ghost region leads to less frequent message exchanges but does not reduce the total amount of data transferred in the network because the payload for each message increases as well. It has been shown to be insignificant in multi-node scenarios due to the slower inter-node communication [21]. Therefore, we decided not to include ghost region sizes/update frequencies as a tuning parameter in our code generator and auto-tuning schemes. For DP stencils, their performance is no better than [5] due to limitations in shared 


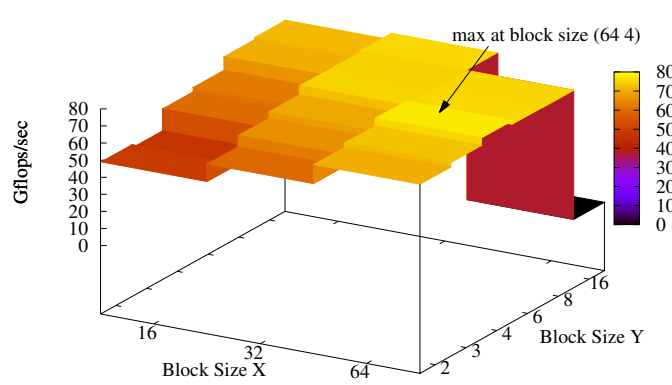

(a) Find Optimal Block Sizes

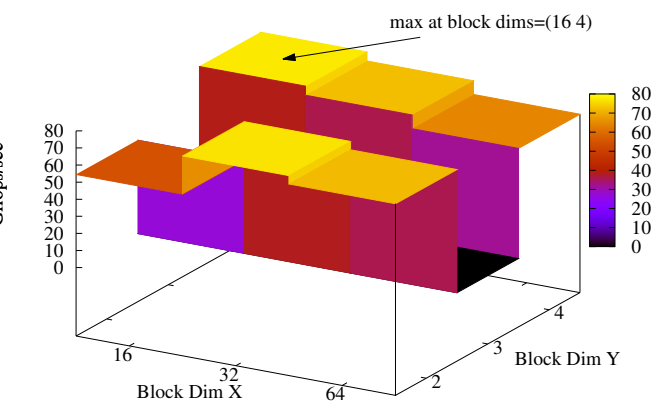

(b) Find Optimal Block Dims, Fixed Block Size at (64,

Figure 10: C2050 27-Point Stencil (DP)

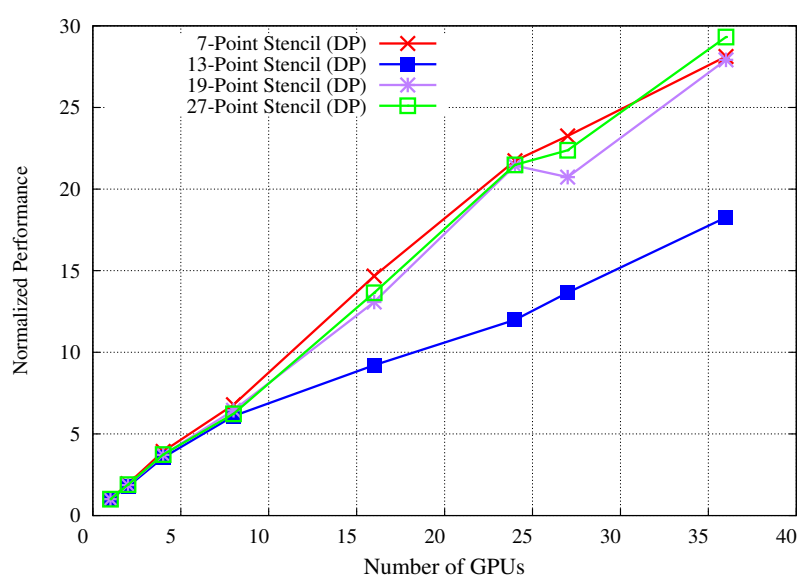

(a) C2050 cluster

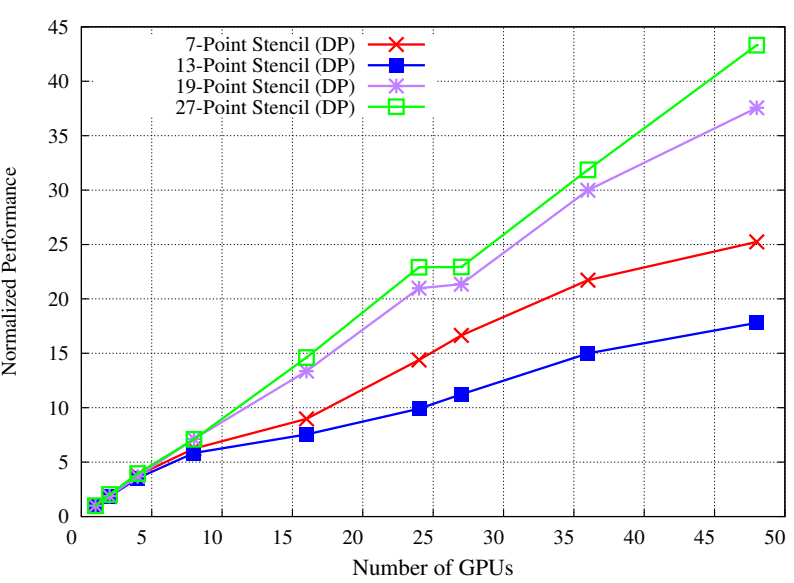

(b) GTX 480 cluster

Figure 11: Weak Scaling of DP Stencils on GPU Clusters

memory size of the GTX 285.

Unat et al. proposed a compiler framework called Mint using annotated $\mathrm{C}$ as the front-end. It converts stencil computation into $\mathrm{C}$ code using pragmas with several levels of optimized CUDA code [22]. Our DP performance of a 7-point stencil on the C1060 achieves the same GFlops as their hand-written code (28 GFlops). In contrast, auto-generated Mint code with the highest level optimization achieves only 22 GFlops.

Christen et al. [4] and Maruyama et al. [14] proposed two DSLs: Patus and Physis. Patus purely depends on the cache on the Fermi architecture without using any shared memory. Therefore, its autotuning capability is severely limited. Physis currently lacks any auto-tuning scheme, one has to choose block sizes manually. Both report SP performance inferior to ours.

\section{CONCLUSION}

This paper shows that GPU programmability and performance are not mutually exclusive under DSLs. With a DSL specification fed to the front-end, problem descriptions can become very concise and intuitive. Using auto-tuning with run-time profile feedback, optimal tuning points within the parameter search space can be identified. Our framework combines auto-generation and auto-tuning of 3D stencil codes on heterogeneous GPU clusters. We extract a small, selective number of key performance-sensitive parameters and auto-tune them to achieve the best possible performance over a variety of GPUs. Compared to previous work, we manage to keep the programmer's effort to even a lower overhead without significant sacrifice in performance.

\section{REFERENCES}

[1] NVIDIA Cooperation, CUDA Programming Guide.

[2] C. W. Antoine, A. Petitet, and J. J. Dongarra. Automated Empirical Optimization of Software and the ATLAS Project. Parallel Computing, 27:2001, 2000.

[3] B. Catanzaro, A. Fox, K. Keutzer, D. Patterson, B.-Y. Su, M. Snir, K. Olukotun, P. Hanrahan, and H. Chafi. Ubiquitous Parallel Computing from Berkeley, Illinois, and Stanford. IEEE Micro, 30(2):41-55, Mar. 2010.

[4] M. Christen, O. Schenk, and H. Burkhart. PATUS: A Code Generation and Autotuning Framework For Parallel Iterative Stencil Computations on Modern Microarchitectures. In IEEE Intl Parallel and Distributed Processing Symposium (IPDPS), May 2011.

[5] K. Datta, M. Murphy, V. Volkov, S. Williams, J. Carter, L. Oliker, D. Patterson, J. Shalf, and K. Yelick. Stencil Computation Optimization and Auto-tuning on State-of-the-art Multicore Architectures. In Proceedings of the 2008 ACM/IEEE conference on Supercomputing, SC '08, pages 4:1-4:12, Piscataway, NJ, USA, 2008. IEEE Press. 
[6] M. Frigo. A Fast Fourier Transform Compiler. SIGPLAN Not., 39:642-655, April 2004.

[7] P. Guo and L. Wang. Auto-Tuning CUDA Parameters for Sparse Matrix-Vector Multiplication on GPUs. Computational and Information Sciences, International Conference on, 0:1154-1157, 2010.

[8] J. L. Gustafson. Reevaluating Amdahl's law. Commun. ACM, 31:532-533, May 1988.

[9] http://www.khronos.org/opencl. OpenCL.

[10] W.-m. Hwu, S. Ryoo, S.-z. Ueng, J. H. Kelm, I. Gelado, S. S. Stone, R. E. Kidd, S. S. Baghsorkhi, A. A. Mahesri, S. C. Tsao, N. Navarro, S. S. Lumetta, M. I. Frank, and S. J. Patel. Implicitly Parallel Programming Models for Thousand-core Microprocessors. In Proceedings of the 44th annual Design Automation Conference, pages 754-759, New York, NY, USA, 2007. ACM.

[11] S. Kamil, C. Chan, L. Oliker, J. Shalf, and S. Williams. An Auto-Tuning Framework for Parallel Multicore Stencil Computations. In In IEEE Intl Parallel and Distributed Processing Symposium (IPDPS), 2010.

[12] Y. Li, J. Dongarra, and S. Tomov. A Note on Auto-tuning GEMM for GPUs. In Proceedings of the 9th International Conference on Computational Science: Part I, ICCS '09, pages 884-892, Berlin, Heidelberg, 2009. Springer-Verlag.

[13] Z. Li and Y. Song. Automatic Tiling of Iterative Stencil Loops. ACM Trans. Program. Lang. Syst., 26:975-1028, November 2004.

[14] N. Maruyama, T. Nomura, K. Sato, and S. Matsuoka. Physis: An Implicitly Parallel Programming Model for Stencil Computations on Large-Scale GPU-Accelerated Supercomputers. 2011.

[15] S. Matsuoka, T. Aoki, T. Endo, A. Nukada, T. Kato, and A. Hasegawa. GPU Accelerated Computing from Hype to Mainstream, the Rebirth of Vector Computing. In Journal of Physics: Conference Series 180, 2009.
[16] J. Meng and K. Skadron. Performance Modeling and Automatic Ghost Zone Optimization for Iterative Stencil Loops on GPUs. In Proceedings of the 23rd international conference on Supercomputing, ICS '09, pages 256-265, New York, NY, USA, 2009. ACM.

[17] P. Micikevicius. 3D Finite Difference Computation on GPUs using CUDA. In Proceedings of 2 nd Workshop on General Purpose Processing on Graphics Processing Units, GPGPU-2, pages 79-84, New York, NY, USA, 2009. ACM.

[18] A. Nguyen, N. Satish, J. Chhugani, C. Kim, and P. Dubey. 3.5-D Blocking Optimization for Stencil Computations on Modern CPUs and GPUs. In Proceedings of the 2010 ACM/IEEE International Conference for High Performance Computing, Networking, Storage and Analysis, SC '10, pages 1-13, Washington, DC, USA, 2010. IEEE Computer Society.

[19] A. Nukada and S. Matsuoka. Auto-tuning 3-D FFT library for CUDA GPUs. In Proceedings of the Conference on High Performance Computing Networking, Storage and Analysis, SC '09, pages 30:1-30:10, New York, NY, USA, 2009. ACM.

[20] E. Phillips and M. Fatica. Implementing the Himeno benchmark with CUDA on GPU clusters. In International Parallel and Distributed Processing Symposium(IPDPS), Apr 2010.

[21] M. Ripeanu, A. Iamnitchi, and I. Foster. Cactus Application: Performance Predictions in Grid Environments. In In proceedings of European Conference on Parallel Computing (EuroPar) 2001, 2001.

[22] D. Unat, X. Cai, and S. Baden. Mint: Realizing CUDA Performance in 3D Stencil Methods with Annotated C. In Proceedings of the 25th International Conference on Supercomputing (ICS'11), 2011.

[23] R. Vuduc, J. W. Demmel, and K. A. Yelick. OSKI: A library of Automatically Tuned Sparse Matrix Kernels. In Institute of Physics Publishing, 2005. 